\title{
Genomic characteristics of recently recognized Vibrio cholerae El Tor lineages associated with cholera in Bangladesh, 1991-2017
}

\section{Authors:}

Md Mamun Monir ${ }^{1}$, Talal Hossain ${ }^{1}$, Masatomo Morita ${ }^{2}$, Makoto Ohnishi $^{2}$, Fatema-Tuz Johura ${ }^{1}$, Marzia Sultana ${ }^{1}$, Shirajum Monira ${ }^{1}$, Tahmeed Ahmed ${ }^{1}$, Nicholas Thomson ${ }^{3}$, Haruo Watanabe ${ }^{2}$, Anwar Huq ${ }^{4}$, Rita R. Colwell ${ }^{4,5}$, Kimberley Seed ${ }^{6}$, and Munirul Alam ${ }^{1 \S}$.

1. icddr, b, Formerly International Centre for Diarrhoeal Disease Research, Bangladesh, Dhaka, Bangladesh

\section{National Institutes of Infectious Diseases (NIID), Tokyo, Japan}

3. Sanger Institute, Cambridge, UK

4. Maryland Pathogen Research Institute, University of Maryland, USA

5. Johns Hopkins Bloomberg School of Public Health, Baltimore, Maryland, USA

6. University of California, Berkeley, USA

${ }^{\S}$ Corresponding author: Munirul Alam, $\mathrm{PhD}$

Mailing address:

Infectious Diseases Division (IDD)

International Centre for Diarrheal Disease Research, Bangladesh (icddr,b)

68, Shaheed Tajuddin Ahmed Sarani,

Mohakhali, Dhaka 1212,

Bangladesh

Tel: +88-02-9840523-32 Ext. 2433/2490

Fax: $+88-02-8812529$

E-Mail: munirul@icddrb.org 


\section{Abstract: (Words 245)}

37 Comparative genomic analysis of Vibrio cholerae El Tor associated with endemic cholera in 38 Asia revealed two distinct lineages, one dominant in Bangladesh and the other in India. An in depth whole genome study of $V$. cholerae El Tor clinical strains isolated during endemic cholera in Bangladesh (1991 - 2017) included reference genome sequence data obtained online. Core genome phylogeny established using single nucleotide polymorphisms (SNPs) showed $V$. cholerae El Tor strains comprised two lineages, BD-1 and BD-2, which, according to Bayesian phylodynamic analysis, originated from paraphyletic group BD-0 around 1981. BD-1 and BD-2 lineages overlapped temporally but were negatively associated as causative agents of cholera 2004-2017. Genome wide association study (GWAS) revealed 140 SNPs and 31 indels, resulting in gene alleles unique to BD-1 and BD-2. Regression analysis of root to tip distance and year of isolation indicated early BD-0 strains at the base, whereas BD-1 and BD-2 subsequently emerged and progressed by accumulating SNPs. Pangenome analysis provided evidence of gene acquisition by both BD-1 and BD-2, of which six crucial proteins of known function were predominant in BD-2. BD-1 and BD-2 diverged and have distinctively different genomic traits, namely heterogeneity in VSP-2, VPI-1, mobile elements, toxin encoding elements, and total gene abundance. In addition, the observed phage-inducible chromosomal island-like element (PLE1), and SXT ICE elements (ICE ${ }^{\mathrm{TET}}$ ) in BD-2 presumably provided a fitness advantage for the lineage to outcompete BD-1 as the etiological agent of the endemic cholera in Bangladesh, with implications for global cholera epidemiology.

Importance: (150 words)

57 Cholera is a global disease with specific reference to the Bay of Bengal Ganges Delta where Vibrio cholerae $\mathrm{O} 1 \mathrm{El}$ Tor, causative agent of the disease showed two circulating lineages, one dominant in Bangladesh and the other in India. Results of in-depth genomic study of $V$. cholerae associated with endemic cholera during the past 27 years $(1991$ - 2017) indicate emergence and succession of the two lineages, BD-1 and BD-2, arising from a common ancestral paraphylatic

62 group, BD-0, comprising the early strains and short-term evolution of the bacterium in

63 Bangladesh. Among the two $V$. cholerae lineages, BD-2 supersedes BD-1 and is predominant in 64 the most recent endemic cholera in Bangladesh. The BD-2 lineage contained significantly more 
SNPs and indels, and showed richness in gene abundance, including antimicrobial resistance genes, gene cassettes, and PLE to fight against bacteriophage infection, acquired over time. These findings have important epidemic implications at a global scale.

\section{Introduction}

Cholera is a life threatening infectious diarrheal disease caused by Vibrio cholerae serogroups $\mathrm{O} 1$ and $\mathrm{O} 139$ of the Gram-negative gammaproteobacteria $(1,2)$. The global incidence of cholera is estimated to be 2.9 million cases annually with almost 95,000 deaths (3). In 2017, 34 countries reported a total of 1,227,391 cases and 5,654 deaths (4). Seven cholera pandemics have been recognized since 1817. However, limited information is available regarding the etiological agent for the first five pandemics and no isolates of the causative agent are extant. The sixth pandemic, and possibly those earlier were caused by $V$. cholerae $\mathrm{O} 1$ classical biotype, while the ongoing seventh pandemic is caused by $V$. cholerae El Tor biotype and began with displacement of $V$. cholerae classical biotype in Asia in 1961 (5). V. cholerae El Tor was isolated in Africa in the 1970s and Latin America in 1991 where for more than a century there had been no cholera outbreaks (6). In 1992, a V. cholerae non-O1 strain designated V. cholerae O139 Bengal initiated outbreaks of cholera in coastal areas of India and Bangladesh, and subsequently was isolated from patients in several countries of Asia (2). V. cholerae El Tor continues to be the major etiological agent of cholera worldwide.

The severe dehydrating diarrhea characteristic of cholera is associated with several factors, including a toxin and several virulence genes involved in colonization and toxicity and their coordinated expression (1). Cholera toxin (CT) is the virulence factor responsible for secretory diarrhea of cholera and is encoded in the genome of a lysogenic CTX phage. V. cholerae El Tor responsible for the current cholera pandemic harbors the CTX phage classical biotype variant, and the $\operatorname{ctxB}^{\text {cla }}$ : $\operatorname{ctxB}$ genotype $1(\operatorname{ctxB} 1)$ or $\operatorname{ctxB7}(7)$. V. cholerae responsible for the current cholera pandemic has become more virulent by undergoing several shifts in CTX genotype and acquiring virulence-related gene islands (8). Integrative conjugative elements (ICEs) and lysogenic phages are genetic elements that play an important role in the acquisition of virulence, antimicrobial resistance, and heavy metal resistance, which important components of the pathogenicity of $V$. cholerae $(9,10)$. Functions of these elements are important for the pathogen to exert evolutionary advantage and variants can be used as markers of clonal expansion (1). 
Acquisition of mobile genetic elements (MGEs) through horizontal gene transfer (HGT) and propitious chromosomal mutations are significant landmarks for an evolving bacterium (11).

Whole-genome sequencing of $V$. cholerae El Tor strains associated with the seventh cholera pandemic revealed three waves, suggesting independent but overlapping paths for the pathogen to spread globally from the Bay of Bengal estuary where cholera has been endemic at least since 1961 but likely for centuries (5). Intercontinental transmission of $V$. cholerae has been proposed for the 2010 outbreak in Haiti (12). Bangladesh borders on the Bay of Bengal and is considered to be a hotspot of Asiatic cholera where -ca. 100,000 cases and 4,500 deaths are reported each year (13). V. cholerae $\mathrm{O} 1$ responsible for endemic cholera in Bangladesh and India has been found to have undergone genetic changes over time including acquisition of classical biotype attributes in an El Tor background, thereby becoming more successful as a pathogen $(14,15)$. A recent whole-genome analysis of Vibrio cholerae El Tor strains isolated between 2009 and 2016 indicated two distinct lineages exist in Bengal (16). The objective of the study reported here was to investigate $V$. cholerae endemic cholera strains isolated during 1991 to 2017 to understand more completely about emergence and progression of the two lineages in Bangladesh. Virulence and related genomic islands, including toxin and antimicrobial resistance genes differing significantly among the $V$. cholerae $\mathrm{El}$ Tor lineages, were also investigated for potential

112 relevance to emergence of the lineages.

\section{Results}

\section{Phylogenetic analysis}

115

A total of 119 strains were included in the study and their genomes were sequenced using the Illumina platform (MiSeq or HiSeq 2500 sequencer). In addition, 56 strains from our previous study (16) and 17 genomes from the European Nucleotide Archive (17) were used, which are representative of isolates from Bangladesh between 1991 and 2017 (see Table S1 Supplemental Material). Paired-end reads of the 192 genomes were mapped to V. cholerae El Tor N16961 reference strain, a seventh-pandemic V. cholerae O1 El Tor (7PET) strain isolated in Bangladesh in 1975 (18). A total of 1,298 single nucleotide polymorphisms (SNPs) and 413 indels (insertions or deletions) were obtained and, after filtering indels, low call rate, and high-density SNPs, a total of 893 high-quality SNPs were retained for further study. A phylogenetic analysis was 
124

125

126

127

128

129

130

131

132

133

134

135

136

137

138

139

140

141

142

143

144

145

146

147

148

149

150

151

152

153

conducted to construct a tree based on the 893 high-quality SNPs to evaluate the genetic diversity of the Vibrio cholerae $\mathrm{O} 1 \mathrm{El}$ Tor isolates from Bangladesh. A nested hierarchical structure in the phylogenetic tree was observed, with all but four of the strains isolated between 1999 and 2017 clustering into two major clades, BD-1 ( $n=76)$ and BD-2 $(n=105)$, shown in green and red, respectively. The remaining strains formed paraphyletic group BD-0 (n=11) (Fig. 1A, in blue). Except for three strains isolated in 2012 that formed a sub-clade, BD-0 consisted mostly of strains isolated earlier between 1991 and 2000. Dates of isolation of common ancestors of the lineages were inferred using Bayesian Markov chain Monte Carlo framework Bayesian Evolutionary Analysis Sampling Trees (BEAST) (19) (see Fig. S1 supplementary material), and a maximum clade credibility (MCC) tree was inferred from the posterior distribution of the best fitting model using program TreeAnnotator tool of the BEAST software package. It was estimated from the MCC tree that the most recent common ancestor (MRCA) of lineage BD-1 was isolated in 1987 (95\% HPD: 1983-1991), and lineage BD-2 in 1997 (95\% HPD: 1994-2000), where HPD stands for height posterior density. Strains of BD-1 and BD-2 shared genome sequences of strains isolated since 1981 (95\% HPD: 1976-1986). The number of SNPs in strains of the two clades is relative to reference $V$. cholerae N16961, which showed strains of BD-0 differed by 107 - 137 SNPs, BD-1 by 123 - 189 SNPs, and BD-2 by 146 - 186 SNPs. An unrooted tree showed SNP diversity among BD-0, BD-1, and BD-2 clades with SNP diversity of BD-2 highest (Fig. 1B). Comparison of isolates in the clades and year of isolation revealed clonal aggregation within the dominant clade and strong temporal signature. Strains of BD-1 and BD-2 were found to be temporally spread but simultaneously isolated during the periods of 2004 2011, 2012, 2014 - 2016 (Fig. 1C, Table S2 supplemental material). Strains of BD-1 were mainly isolated during 2004-2011 (66.3\%, $\mathrm{n}=65)$ while strains of BD-2 were isolated during those years in fewer numbers $(33.7 \%, \mathrm{n}=33)$ except 2009 when BD-2 strains were dominant (93.33\%, n=14) (see Table S1 in supplementary material). The following years, from 2012 to 2017, showed BD-2 strains to be dominant $(73.5 \%, \mathrm{n}=72)$ and BD-1 strains the minority $(10.2 \%$, $\mathrm{n}=10)$.

\section{Genetic variants associated with the clades}

Associations between lineages and the genetic variants was studied using 1298 SNPs and 413 indels, identified by aligning raw reads against $V$. cholerae N16961 reference genome. Variant 
annotation using SnpEff (20) showed that among the 1298 SNPs, there were 337 synonymous, 613 nonsynonymous, and 348 variants on intergenic regions (Fig. 2A-C, see Table S2 in the supplemental materials). Moreover, of 413 indels, there were 238 frameshift-variants, 107 variants on intergenic regions, and 68 other types of variants (Fig 2D-F, Table S2). Most of the identified SNPs and indels were located in the protein-coding region, many of which function to change the form of a protein. By plotting distribution of SNP types and indel variants for BD-0 $(n=11), B D-1(n=76)$, and BD-2 $(n=105)$, it was observed that strains of the clades accumulated SNPs and indels. Strains of BD-2 accumulated more SNPs and indels, increasing genetic distance from BD-0 and BD-1 (Fig. 1B, Fig. 2) and suggesting evolution was occurring when compared with reference $V$. cholerae O1 N16961.

Fisher exact test (21) was performed for association analysis between genetic variants and the clades BD-1 and BD-2. Association analysis showed that 140 SNPs and 31 indels had a genomewide significant association $\left(p<6.40 \times 10^{-9}\right)$ with BD-1 and BD-2. Among the 140 SNPs were 25 synonymous variants, 53 missense variants, 2 stop gain variants, and 60 variants on intergenic regions (Table S3 and Fig. S2 in supplementary material). It was discovered that 21 SNP missense mutations were present in genes with known functions in more than $80 \%$ of BD2 strains, resulting in mutant proteins (Table 1). However, there were only seven missense mutations were found in genes with known functions in more than $80 \%$ of BD1 strains. Genotype and frequency of 140 significantly associated SNPs, number of SNPs by year of isolation, and root to tip distance, showed significant genetic differences between BD-1 and BD2 (Fig. 3). The number of core genome SNPs by year of isolation was analyzed to detect temporal SNP accumulation patterns of the clades. The number of core genome SNPs did increase over time for both BD-1 and BD-2 (Fig. 3B). Moreover, root-to-tip regression analysis indicated a steady increase in SNP divergence among the strains of the two clades over time (Fig. 3C). Miami plot for frequency of alternative alleles of the 140 significant SNPs showed BD-2 strains had accumulated more clade specific SNPs, notably in chromosome-2 compared to BD-1 (Fig. 3D).

\section{Relative gene abundance}

Pangenome analysis was done using Roary to investigate differences in core and pan genes among the strains of BD-0, BD-1, and BD-2. Roary classified the identified functional genes into 
four categories: (i) core genes, present in $99-100 \%$ of the strains; (ii) softcore genes, present in 95-99\% of the strains; (iii) shell genes, present in 15-95\% of the strains; and (iv) cloud genes, present in less than 15\% of the strains (22). Pangenome analysis revealed significant differences in overall gene composition among the clades (Fig 4A). According to the definition of core genes in pangenome analysis, the number of core genes largely varied among BD-0, BD-1, and BD-2 (see Table S4 in supplementary material). Similarly, the number of soft-core genes was also varied. BD-0 is a group of close relatives with a larger genetic distance relative to BD-1 and BD2. All BD-0 strains and more than $95 \%$ of the BD-1 and BD-2 strains had 1102 common genes (see Table S5A in supplementary material) most having known function. About 10\% of BD-2 strains had 44 unique genes of which six encoding crucial proteins of known function were found in more than $90 \%$ of the BD-2 strains. Those genes are: tetracycline repressor protein (tetR), tetracycline resistance protein (tetA), type-I restriction enzyme EcoKI M protein (hsdM), type-I restriction enzyme EcoR124II R protein (hsdR), Mrr restriction system protein (mrr), and 5-methylcytosine-specific restriction enzyme B (mcrB) (see Table S5B in supplementary material). In addition, methyl-accepting chemotaxis protein (CtpH) and group_10030 virulence proteins were exclusively found in $60 \%$ and $65 \%$ of BD-2 strains, respectively. By contrast, about $5-15 \%$ of the BD-1 strains carried 19 genes that were unique for them (see Table S5C in supplementary material). Three genes common to all BD-0 strains were not detected in BD-2 and were present only in 1-2 of the BD-1 strains.

Next, we conducted Pan-GWAS to identify clade-specific genes by considering gene presence and absence as the explanatory variable and defined lineage groups as the response variable. A total of 92 genes were significantly ( $\mathrm{p}$-value $<4.98 \times 10^{-6}$ ) associated with BD-0 and BD-1 (see Table S6A in supplementary material). Of these, 62 genes were identified in 54-73\% of BD-0 but not in BD-1 strains. Of 164 genes associated with BD-0 and BD-2, 46 were found in more than $73 \%$ of BD-2, but not in BD-0 strains (see Table S6B in supplementary material). In addition, 66 genes were found in more than $45 \%$ of BD-0, but not in BD-2 strains. Of 143 genes associated with BD-1 and BD-2 (see Table S6C in supplementary material), 29 were found in more than $76 \%$ of BD-1, but not in BD-2 strains. Again, 47 genes were found in 22-97\% of the BD-2, but not in BD-1 strains. These results provide evidence that strains of BD-1 and BD-2 diverged and evolved as two lineages by accumulating genes, after originating from common ancestor BD-0. 


\section{Pathogenicity islands and phage inducible chromosomal island like elements}

216

217

218

219

220

221

222

223

224

225

226

227

228

229

230

231

232

233

234

235

236

237

238

239

240

241

242

243

$V$. cholerae strains included in this study were further examined by targeting the pandemic and pathogenicity islands namely VSP-1, VSP-II, VPI-1, and VPI-2, including the phage inducible chromosomal island like elements (PLE). Based on the extent of detected regions compared to $V$. cholerae N16961, five variants of VSP-II (variants 1-5 of the wild type) as reported in our recent study (16), and one variant of VPI-1 (variant 1 of the wild type) were observed (Fig 5). $V$. cholerae El Tor strains differed in type of VSP-II and VPI-1 variants. BD-0 had wild type of VSP-II, as in reference El Tor N16961 strain. Most BD-1 strains (except two) had variant-4 VSP-II, with partial deletion in VC_495 and complete deletion in VC_496 to VC_512, and BD2 strains carried three VSP-II variants of which ca. $73 \%$ had variant-2 VSP-II with partial ORF VC_495 deletion, and complete VC_496 to VC_500 deletion, which appeared consistent with our prior study (16). BD-0 and BD-1 harbored wild type of VPI-1, whereas most of the BD-2 strains (102 of 105 strains) had variant VPI-1 with complete deletion of VC_819 to VC_820 ORFs; and partial deletion in VC_821. All BD-0 strains, and 66 of 76 BD-1 strains lacked PLE (see Tables S1 and S7 in supplementary material), while PLE2 was found in ten BD-1 strains isolated in 2007 possessing the $\operatorname{ctxB} 1$ genotype and one in 2005. Interestingly, most of the BD-2 strains (83 of 103) carried PLE1, but the rest lacked PLE. Thus, BD-2 lineage strains associated with recent Bangladesh endemic cholera are variant-3 VSP-II, variant VPI-1, and the majority possesses PLE1.

\section{Variations in SXT/R391 and important genes}

Although differences in SXT/R391, ctxB, gyrA, rtxA, and parC across two lineages (BD-1, analogue of lineage-2; BD-2, analogue of lineage-1) were investigated in our recent study (16), these important genetic elements were rechecked to draw overall conclusions for all strains included in this investigation. Moreover, variation in ToxR binding repeats were checked across strains of different lineages. Integrative and conjugative elements (ICEs) were targeted from whole-genome sequences by aligning raw reads or contigs with five publicly available sequences of the ICE element (Accession ID: GQ463140.1, GQ463141.1, GQ463142.1, MK165649.1, and MK165650.1). Nucleotide blast was used to match extracted sequences with ICE element sequences and typed based on highest bit score. Four strains of BD-0 blast search yielded high 
244 bit scores when aligned with ICE ${ }^{\mathrm{GEN}}$ (MK165650.1), ICEVchInd5 (GQ463142.1), or

245 ICEVchBan5 (GQ463140.1). Bit scores were highest for the other BD-0 strains when aligned 246 with ICE ${ }^{\mathrm{TET}}$ (Accession ID: MK165649.1), which has genomic characteristics similar to 247 ICEVchVhn2255 (Accession ID: KT151660). For all BD-1 strain bit scores were high when 248 aligned with ICE ${ }^{\mathrm{GEN}}$, ICEVchInd5, or ICEVchBan5, and for BD-2 strains bit scores were highest when aligned with $\mathrm{ICE}^{\mathrm{TET}}$, which is consistent with our previous results. All BD-1 and BD-2 strains contained mutant gyrA with an amino acid alteration Ser83Ile, whereas 99 (94.28 percent) of the 105 BD-2 strains exhibited Asp660Glu, which was not present in BD-1 or BD-0, also supporting our previous findings.

V. cholerae $\mathrm{O} 1 \mathrm{El}$ Tor strains in this study were CTX positive, and each carried a single copy of СТХФ with a particular $c t x B$ genotype. Three variants, $c t x B 1$ (classical genotype), $c t x B 3$ (typical El Tor genotype), and $\operatorname{ctxB7}$ (Haitian variant), of the cholera toxin gene were detected and found associated with the clades (Fig. 1A). Similar to previous findings, all BD-2 strains had ctxB1 genotype, majority of BD-1 strains had $c t x B 7$ genotype, and all but two BD-1 strains possessed rtxA that differed from El Tor reference N16961 by a single SNP at position 13602 of 1563748 bp (NCBI Accession ID: NC 002505.1), corresponding to rtxA allele 4 (23). However, in this study it was observed that early BD-1 strains had the $\operatorname{ctxBl}$ genotype, and over time gained the ctxB7 genotype.

A prior study showed that, Kolkata strains had four heptad repeats (TTTTGAT), whereas Haitian strains had five heptad repeats (24). All BD-0 strains had four heptad repeats (Table S1), while most BD-1 strains (93.4\%; $n=71)$ had four repeats, and only 5.3\% ( $n=4)$ strains had five repeats. As a result, the majority of BD-1 strains with $\operatorname{ctxB7}$ genotypes differed from Haitian strains in ToxR binding repeats. BD-2 strains had more diversity in ToxR binding repeats with $59.0 \%(n=62)$ carrying heptad repeats, $24.8 \%(n=26)$ five repeats, and $16.2 \%(n=17)$ three repeats.

\section{Discussion}

270 Vibrio cholerae biotype El Tor, the causative agent of the $7^{\text {th }}$ cholera pandemic has increased 271 transmissibility and is more virulent than classical biotype $(14,15)$. The 7 th pandemic strains of 272 cholera circulating in Asia comprises two El Tor clades, one dominant in Bangladesh and the 
273 other in India (16). Genomic analyses that included additional strains and publicly available genome sequences of wave- 2 and wave- 3 strains $(6,12)$ provide a detailed view of longitudinally and temporally representative $V$. cholerae clades associated with endemic cholera in Bangladesh over a period of 27 years (1991 - 2017). The results provide new insights potentially interpretable as origin and progression, based on differences in SNPs, indels, and gene acquisition, including antibiotic resistance cassettes in BD-1 and BD-2, the latter having gained ascendency and dominance as the agent of Bangladesh endemic cholera.

Results of whole genome sequencing (16), combined with additional genome sequence data for $V$. cholerae El Tor isolates of Bangladesh endemic cholera, allowed identification of two lineages, designated BD-1 and BD-2. The two clades appear to have originated from a common ancestor of paraphyletic group BD-0, as early as 1981 (95\% HPD: 1976-1986). According to A. Mutreja et al. (12), seven strains of BD-0 isolated between 1991 and 2000 represent wave-2 strains, and only one strain isolated in 1994, wave-3 with a most recent common ancestor (MRCA) for BD-1 and BD-2. The BD-1 and BD-2 clades may belong to wave-3. Although BD0 consisted of predominantly of wave- 2 strains, three sequenced strains isolated in 2012 shared a wave-2-like genetic background (6), suggesting wave-2 strains may have already been present. Almost all wave-3 strains from a previous study (12) grouped with strains belonging to BD-1. Consistent with results of a previous study (16), significant differences were noted between BD1 and BD-2, which varied in temporal predominance as the causal agent of Bangladesh endemic cholera. Most ( $\mathrm{n}=62 ; 82$ percent) BD-1 strains had been isolated between 2007 and 2012, with predominance during that time. Between 2005 and 2017, 105 strains belonging to BD-2 were reported, with 97 obtained between 2009 and 2017, implying BD-2 association with recent Bangladesh endemic cholera until 2017. Phylodynamic analysis using BEAST (19) revealed strains of BD-1 had been isolated in Bangladesh roughly ten years before BD-2 strains (see, Fig.

297 S1 in supplementary material), and previously identified as Asian lineage -2 and Asian lineage-1, respectively (16).

BD-1 and BD-2 strains appear to have advanced by accumulating different SNPs and indels. Fisher exact test (21) identified 140 SNPs and 31 indel differences between BD-1 and BD-2, resulting in gene alleles unique to them (Fig 3). The majority of the SNPs and indels were components of protein coding genes, suggesting a possible crucial role in their adaption in 
Bangladesh. Regression analysis of the number of SNPs and year of isolation suggested that both clades consistently accumulated SNPs over time, implying evolution in response to environmental selective pressure.

Pangenome analysis using Roary (22) provided evidence of gene acquisition by strains of the clades. A recent study of $V$. cholerae $\mathrm{O} 1$ strains isolated in Pakistan found evidence of gene acquisition, where the number of core and accessory genes varied among different lineages (25). According to results of the analysis reported here, the number of core and accessory genes varied significantly among strains of BD-0, BD-1, and BD-2 in Bangladesh (Fig. 4A). The Pan-GWAS approach helped identify genes unique for each clade which could be considered contributing to virulence and/or niche adaptation (26).

Phage inducible chromosomal island like elements (PLE) protect $V$. cholerae populations from ICP1 infection by acting as an abortive infection system (27). In this study, the observed predominance in BD-2 of PLE1, not found in BD-0 and BD-1, could have provided a selective advantage for the lineage over BD-1, establishing dominance as an etiological agent of endemic cholera in Bangladesh in recent years.

Two BD-0 strains carried CTX phage with $\operatorname{ctxB3}$, while other strains carried CTX phage with typical $\operatorname{ctxB1}$. Strains at the base of BD-1 had CTX with $\operatorname{ctxB1}$ isolated before 2007 and comprised multiple clusters. Moreover, CTX phage of all BD-2 strains contained classical $c t x B 1$. A mutation in rtxA creating a premature stop codon disabled toxin function in emerging $V$. cholerae El Tor strains bearing $\operatorname{ctxB1}(24)$. As in the classical strains, altered El Tor pandemic strains eliminated $\operatorname{rtxA}$ after acquiring classical $\operatorname{ctx} B$. In this study, BD-0 and BD-2 strains contained the wild-type $r t x A$ allele 1 (Fig. 3A) described by Dolores and Satchell (23). None contained deletions in $r s t B$ gene when reads were compared to $V$. cholerae N16961 reference genome, indicating $r s t B$ of Bangladesh $V$. cholerae $\mathrm{O} 1 \mathrm{El}$ Tor isolates does not resemble that of the Haitian outbreak isolates that have been analyzed.

ToxR is a global transcriptional regulator of virulence gene expression and this repeated sequence is required for ToxR binding and activation of the $\operatorname{ctx} A B$ promoter. The ToxR-binding site is located immediately upstream of $\operatorname{ctx} A B$ and the affinity of ToxR binding is influenced by the repeat sequences (28). The presence of an increased number of ToxR binding repeats located 
between zot and $\operatorname{ctxA}$ has been hypothesized to correlate with a severe form of cholera (28). In this study, variation was detected in the number of ToxR binding repeats (TTTTGAT) among sequences of the $V$. cholerae El Tor isolates. All BD-0 strains had four heptad repeats observed in $93.4 \%$ of BD-1 and $59 \%$ of BD-2 strains. For BD-2 strains, however, greater variation was observed in ToxR binding repeats as ca. 24.8\% ( $n=26)$ of BD-2 strains contained five heptad repeats, whereas $16.2 \%(n=17)$ had three heptad repeats, suggesting robustness of the clade.

Targets of quinolones are type II topoisomerases of DNA gyrase, a heterotetramer composed of two A and two B subunits, encoded by gyrA and gyrB genes respectively (29). It was observed that all BD-1 and BD-2 strains had a common mutation Ser83 to Ile in gyrA, while 94.29\% (99/105) BD-2 had an additional mutation Asp660 to Glu. Furthermore, 87\% (66/76) of BD-1 strains exhibited a mutation Ser85 to Leu parC, whereas all BD-2 strains (105/105) had this mutation. In Haitian $V$. cholerae strains, gyrA and parC genes had two point mutations: Ser83 to Ile in gyrA and Ser85 to Leu in parC. Both are linked to quinolone resistance in $V$. cholerae strains associated with recent cholera outbreaks in India, Nigeria, and Cameroon (30).

SXT/R391 family ICEs are transferable elements associated with antimicrobial resistance in $V$. cholerae (31). The SXT-ICE regions of the isolates included in this study, were compared with five sequences of the elements to the type SXT/R391 family ICEs belonging to strains associated with cholera ( $V$. cholerae O1 and O139) $(9,32)$. Four BD-0 strains exhibited ICE elements similar to ICE ${ }^{\mathrm{GEN}}$, ICEVchInd5 or ICEVchBan5, whereas the rest had ICE elements similar to $\mathrm{ICE}^{\mathrm{TET}}$. Interestingly, ICE elements of BD-1 strains included $\mathrm{ICE}^{\mathrm{GEN}}$, ICEVchInd5 or ICEVchBan5-like ICE elements, whereas BD-2 strains differed completely from the others, with only $\mathrm{ICE}^{\mathrm{TET}}$-like ICE elements.

The results of the study reported here included BD-1 and BD-2 isolated during the Bangladesh endemic cholera of 2004 onwards and that, while existing together, with each subsequent year they exhibited different dominance. BD-2 diverged, while retaining the ability to produce multifunctional-autoprocessing repeats-in-toxin (MARTX) and acquiring SXT element ICE ${ }^{\mathrm{TET}}$ containing tetracycline resistance genes. This observation hints at a selective advantage of BD-2 strains over BD-1 strains for robustness. It is evident from results of the analyses that BD-1 and BD-2 differ significantly, owing to gene composition and SNPs and may have evolved independently due to selection pressures. The use of antibiotics, including tetracycline, can exert 
362

363

364

365

366

367

368

369

370

371

372

373

374

375

376

377

378

379

380

381

382

383

384

385

386

387

388

389

selection pressure in evolution $(16,33)$, while strains stopping to produce MARTX along with other variations in the genome might provide a selective advantage. According to suggestions from studies of the dynamics of $V$. cholerae, immunocompetence of the host against $V$. cholerae strains may contribute to the dynamics of $V$. cholerae, hence produce an effect from interaction with humans in selection and cannot be ruled out (34).

Cholera globally is influenced by thriving populations of $V$. cholerae occurring naturally in the Ganges Delta of Bay of Bengal (GDBB) $(1,2,5,14)$. Overall results presented here suggest means of emergence and progression of the two clades in evolution from a progenitor $V$. cholerae El Tor initiating the seventh pandemic in Asia (5) and reflecting short-term evolution of $V$. cholerae El Tor associated with Bangladesh endemic cholera in the GDBB $(14,31)$. BD-2 is concluded to have emerged relatively recently and evolves by acquiring SNPs over time. Also, BD-2 strains showed diversity in indels, possessing SXT/R391 family ICE-elements, PLE1, tetR, and several other important genetic elements, and predominantly associated with recent Bangladesh endemic cholera. As is apparent from our results, BD-1 appears to be an analogue of a previously reported lineage 2 from Asia, the major causative agent of cholera in India, Yemen, and Haiti (16). In contrast, BD-2 strains of the present study appear to be an analogue of Asian lineage 1, which successfully outcompeted BD-1 (Asian lineage 2) and established predominance as an etiological agent of cholera in an historical hotspot of the disease, Bangladesh. It can be concluded that this is a reflection of robustness of BD-2 as an epidemic clone emerging locally with potential to transmit globally, and underscoring the need to track the two successful $V$. cholerae El Tor clades.

\section{Materials and Methods}

\section{Bacterial isolates}

A total of $119 \mathrm{~V}$. cholerae O1 strains from the icddr,b collection of strains isolated in Bangladesh between 2004 and 2017 (see Table S1 in supplementary material) were sequenced. Paired-end Illumina short reads for the isolated strains were generated (150 bp, $150 \mathrm{bp}$ ) using MiSeq or Hiseq 2500 sequencer as described in our recent study (16). Publicly available paired-end raw reads of 17 strains isolated in Bangladesh between 1991 and 2007 (see study flow chart Fig. S3 
in supplementary material) and 56 strains from our recent study (16) were included in the analysis.

\section{Genome assembly and gene annotation}

An ultra-fast FASTQ preprocessor implemented in FASTP (35), was used to inspect raw pairedend reads and filter bad ligation or adapter parts. De novo genome assembly implemented in VelvetOptimizer (36) was used to build contigs by optimizing the parameter N50, a metric for assessing contiguity of an assembly. The bacterial genome annotation tool, Prokka (37), was used for whole-genome gene annotation. ResFinder (38) was used to find the antimicrobial resistant gene profiles for all of the strains.

\section{SNP identification and phylogenetic analysis}

Bowtie2 (39) was used to align high-quality reads with reference genome sequence of $V$. cholerae N16961 El Tor (NCBI Accession ID: NC_002505.1 and NC_002506.1) for variant calling. Samtools (40) and Bcftools (41) were used to call genome variants. A maximumlikelihood phylogeny was inferred on an alignment of concatenated SNPs evenly distributed across non-repetitive, non-recombinant core genome using IQ-TREE v1.6.1 (42). Trees were visualized in FigTree v1.4.3 (http://tree.bio.ed.ac.uk/ software/figtree/) or Interactive Tree of Life online tool (43).

\section{Bayesian phylogenetic inference}

The Bayesian Evolutionary Analysis Sampling Trees (BEAST) v.2.4.4 software package (19) was used for temporal analysis to estimate divergence date of $V$. cholerae $\mathrm{O} 1$ isolates in Bangladesh. The date of isolation of each strain was used as tip data. A random clock model was implemented using Markov Chain Monte Carlo (MCMC) chains run for 100 million generations with $10 \%$ burn-in and sampled every 1000 generations. A GTR nucleotide substitution model was used. Tree data were summarized using TreeAnotator, a tool of BEAST software package, to generate the maximum clade credibility tree.

\section{Pangenome analysis}


417 A pan-genome was constructed using Roary (22) from annotated assemblies of the sample set 418 with percentage protein identity of 95\%. The protein sequences were first extracted and 419 iteratively pre-clustered with cd-hit (version 4.6) down to 98\% identity. An all against all blast 420 (version 2.2.31) was performed on the remaining non-clustered sequences and a single 421 representative sequence from each cd-hit cluster was selected. The data were used by MCL (44) 422 (version 11-294) to cluster the sequences. The preclusters and MCL clusters were merged and 423 paralogs split by inspecting the conserved gene neighborhood around each sequence (5 genes on 424 either side). Each sequence for each cluster was independently aligned using PRANK (45) 425 (version 0.140603) and combined to form a multi-FASTA alignment of the core genes. 426 Sequences of SXT elements were compared with $\mathrm{ICE}^{\mathrm{GEN}}$ and $\mathrm{ICE}^{\mathrm{TET}}$ using BRIG 0.95 with $70 \%$ 427 BLAST identity (46).

Acknowledgements. This work was supported in part by icddr,b, National Institutes of Infectious Diseases (NIID), Tokyo, and the Research Program on Emerging and Re-emerging Infectious Diseases (JP21fk0108139) from the Japan Agency for Medical Research and Development (AMED). Authors acknowledge icddr,b hospital and laboratory staffs for their support. icddr,b gratefully acknowledges the following donors for providing unrestricted support: Governments of the People's Republic of Bangladesh, Global Affairs Canada (GAC), Swedish 435 International Development Cooperation Agency (Sida), and the Foreign Commonwealth \& 436 Development Office (FCDO), UK. All the authors read and approved the final manuscript. None 437 declared conflicts of interest.

\section{Reference}

1. Reidl J, Klose KE. 2002. Vibrio cholerae and cholera: out of the water and into the host. FEMS Microbiol Rev 26:125-39.

2. Albert MJ, Siddique A, Islam M, Faruque A, Ansaruzzaman M, Faruque S, Sack RB. 1993. Large outbreak of clinical cholera due to Vibrio cholerae non-01 in Bangladesh. The Lancet 341:704.

3. Ramamurthy T, Das B, Chakraborty S, Mukhopadhyay AK, Sack DA. 2019. Diagnostic techniques for rapid detection of Vibrio cholerae 01/0139. Vaccine doi:10.1016/j.vaccine.2019.07.099.

4. Clemens JD, Nair GB, Ahmed T, Qadri F, Holmgren J. 2017. Cholera. The Lancet 390:1539-1549.

5. Hu D, Liu B, Feng L, Ding P, Guo X, Wang M, Cao B, Reeves PR, Wang L. 2016. Origins of the current seventh cholera pandemic. Proc Natl Acad Sci U S A 113:E7730-E7739. 
6. Domman D, Quilici ML, Dorman MJ, Njamkepo E, Mutreja A, Mather AE, Delgado G, MoralesEspinosa R, Grimont PAD, Lizarraga-Partida ML, Bouchier C, Aanensen DM, Kuri-Morales P, Tarr CL, Dougan G, Parkhill J, Campos J, Cravioto A, Weill FX, Thomson NR. 2017. Integrated view of Vibrio cholerae in the Americas. Science 358:789-793.

7. Kim EJ, Lee D, Moon SH, Lee CH, Kim SJ, Lee JH, Kim JO, Song M, Das B, Clemens JD, Pape JW, Nair GB, Kim DW. 2014. Molecular insights into the evolutionary pathway of Vibrio cholerae 01 atypical El Tor variants. PLoS Pathog 10:e1004384.

8. Rashid MU, Rashed SM, Islam T, Johura FT, Watanabe H, Ohnishi M, Alam M. 2016. CtxB1 outcompetes CtxB7 in Vibrio cholerae 01, Bangladesh. J Med Microbiol 65:101-103.

9. Wozniak RA, Fouts DE, Spagnoletti M, Colombo MM, Ceccarelli D, Garriss G, Dery C, Burrus V, Waldor MK. 2009. Comparative ICE genomics: insights into the evolution of the SXT/R391 family of ICEs. PLoS Genet 5:e1000786.

10. Faruque SM, Mekalanos JJ. 2003. Pathogenicity islands and phages in Vibrio cholerae evolution. Trends Microbiol 11:505-10.

11. Murphy RA, Boyd EF. 2008. Three pathogenicity islands of Vibrio cholerae can excise from the chromosome and form circular intermediates. J Bacteriol 190:636-47.

12. Mutreja A, Kim DW, Thomson NR, Connor TR, Lee JH, Kariuki S, Croucher NJ, Choi SY, Harris SR, Lebens M, Niyogi SK, Kim EJ, Ramamurthy T, Chun J, Wood JL, Clemens JD, Czerkinsky C, Nair GB, Holmgren J, Parkhill J, Dougan G. 2011. Evidence for several waves of global transmission in the seventh cholera pandemic. Nature 477:462-5.

13. Islam MT, Clemens JD, Qadri F. 2018. Cholera Control and Prevention in Bangladesh: An Evaluation of the Situation and Solutions. J Infect Dis 218:S171-S172.

14. Nair GB, Qadri F, Holmgren J, Svennerholm AM, Safa A, Bhuiyan NA, Ahmad QS, Faruque SM, Faruque AS, Takeda Y, Sack DA. 2006. Cholera due to altered El Tor strains of Vibrio cholerae 01 in Bangladesh. J Clin Microbiol 44:4211-3.

15. Taneja N, Mishra A, Sangar G, Singh G, Sharma M. 2009. Outbreaks caused by new variants of Vibrio cholerae $01 \mathrm{El}$ Tor, India. Emerg Infect Dis 15:352-4.

16. Morita D, Morita M, Alam M, Mukhopadhyay AK, Johura F-t, Sultana M, Monira S, Ahmed N, Chowdhury G, Dutta S. 2020. Whole-Genome Analysis of Clinical Vibrio cholerae 01 in Kolkata, India, and Dhaka, Bangladesh, Reveals Two Lineages of Circulating Strains, Indicating Variation in Genomic Attributes. Mbio 11.

17. Leinonen R, Akhtar R, Birney E, Bower L, Cerdeno-Tarraga A, Cheng Y, Cleland I, Faruque N, Goodgame N, Gibson R, Hoad G, Jang M, Pakseresht N, Plaister S, Radhakrishnan R, Reddy K, Sobhany S, Ten Hoopen P, Vaughan R, Zalunin V, Cochrane G. 2011. The European Nucleotide Archive. Nucleic Acids Res 39:D28-31.

18. Baddam R, Sarker N, Ahmed D, Mazumder R, Abdullah A, Morshed R, Hussain A, Begum S, Shahrin L, Khan Al, Islam MS, Ahmed T, Alam M, Clemens JD, Ahmed N. 2020. Genome Dynamics of Vibrio cholerae Isolates Linked to Seasonal Outbreaks of Cholera in Dhaka, Bangladesh. mBio 11.

19. Suchard MA, Lemey P, Baele G, Ayres DL, Drummond AJ, Rambaut A. 2018. Bayesian phylogenetic and phylodynamic data integration using BEAST 1.10. Virus Evol 4:vey016.

20. Cingolani P, Platts A, Wang le L, Coon M, Nguyen T, Wang L, Land SJ, Lu X, Ruden DM. 2012. A program for annotating and predicting the effects of single nucleotide polymorphisms, SnpEff: SNPs in the genome of Drosophila melanogaster strain w1118; iso-2; iso-3. Fly (Austin) 6:80-92.

21. Raymond M, Rousset F. 1995. An exact test for population differentiation. Evolution:1280-1283.

22. Page AJ, Cummins CA, Hunt M, Wong VK, Reuter S, Holden MT, Fookes M, Falush D, Keane JA, Parkhill J. 2015. Roary: rapid large-scale prokaryote pan genome analysis. Bioinformatics 31:3691-3. 
23. Dolores J, Satchell KJ. 2013. Analysis of Vibrio cholerae genome sequences reveals unique rtxA variants in environmental strains and an rtxA-null mutation in recent altered El Tor isolates. mBio 4:e00624.

24. Ghosh P, Naha A, Pazhani GP, Ramamurthy T, Mukhopadhyay AK. 2014. Genetic traits of Vibrio cholerae $\mathrm{O} 1$ Haitian isolates that are absent in contemporary strains from Kolkata, India. PLoS One 9:e112973.

25. Zeb S, Gulfam SM, Bokhari H. 2020. Comparative core/pan genome analysis of Vibrio cholerae isolates from Pakistan. Infect Genet Evol 82:104316.

26. Gori A, Harrison OB, Mlia E, Nishihara Y, Chan JM, Msefula J, Mallewa M, Dube Q, Swarthout TD, Nobbs AH, Maiden MCJ, French N, Heyderman RS. 2020. Pan-GWAS of Streptococcus agalactiae Highlights Lineage-Specific Genes Associated with Virulence and Niche Adaptation. mBio 11.

27. Hays SG, Seed KD. 2020. Dominant Vibrio cholerae phage exhibits lysis inhibition sensitive to disruption by a defensive phage satellite. eLife 9:e53200.

28. Pfau JD, Taylor RK. 1996. Genetic footprint on the ToxR-binding site in the promoter for cholera toxin. Mol Microbiol 20:213-22.

29. Hooper DC. 1998. Clinical applications of quinolones. Biochim Biophys Acta 1400:45-61.

30. Hasan NA, Choi SY, Eppinger M, Clark PW, Chen A, Alam M, Haley BJ, Taviani E, Hine E, Su Q, Tallon L, Prosper JB, Furth K, Hoq MM, Li H, Fraser-Liggett CM, Cravioto A, Huq A, Ravel J, Cebula TA, Colwell RR. 2012. Genomic diversity of 2010 Haitian cholera outbreak strains. Proc Natl Acad Sci U S A 109:E2010-7.

31. Weill FX, Domman D, Njamkepo E, Almesbahi AA, Naji M, Nasher SS, Rakesh A, Assiri AM, Sharma NC, Kariuki S, Pourshafie MR, Rauzier J, Abubakar A, Carter JY, Wamala JF, Seguin C, Bouchier C, Malliavin T, Bakhshi B, Abulmaali HHN, Kumar D, Njoroge SM, Malik MR, Kiiru J, Luquero FJ, Azman AS, Ramamurthy T, Thomson NR, Quilici ML. 2019. Genomic insights into the 2016-2017 cholera epidemic in Yemen. Nature 565:230-233.

32. Sarkar A, Morita D, Ghosh A, Chowdhury G, Mukhopadhyay AK, Okamoto K, Ramamurthy T. 2019. Altered Integrative and Conjugative Elements (ICEs) in Recent Vibrio cholerae 01 Isolated From Cholera Cases, Kolkata, India. Front Microbiol 10:2072.

33. Tello A, Austin B, Telfer TC. 2012. Selective pressure of antibiotic pollution on bacteria of importance to public health. Environ Health Perspect 120:1100-6.

34. Chakraborty S, Mukhopadhyay AK, Bhadra RK, Ghosh AN, Mitra R, Shimada T, Yamasaki S, Faruque SM, Takeda Y, Colwell RR, Nair GB. 2000. Virulence genes in environmental strains of Vibrio cholerae. Appl Environ Microbiol 66:4022-8.

35. Chen S, Zhou Y, Chen Y, Gu J. 2018. fastp: an ultra-fast all-in-one FASTQ preprocessor. Bioinformatics 34:i884-i890.

36. Zerbino DR, Birney E. 2008. Velvet: algorithms for de novo short read assembly using de Bruijn graphs. Genome Res 18:821-9.

37. Seemann T. 2014. Prokka: rapid prokaryotic genome annotation. Bioinformatics 30:2068-9.

38. Zankari E, Hasman H, Cosentino S, Vestergaard M, Rasmussen S, Lund O, Aarestrup FM, Larsen MV. 2012. Identification of acquired antimicrobial resistance genes. J Antimicrob Chemother 67:2640-4.

39. Langmead B, Salzberg SL. 2012. Fast gapped-read alignment with Bowtie 2. Nat Methods 9:3579.

40. Li H, Handsaker B, Wysoker A, Fennell T, Ruan J, Homer N, Marth G, Abecasis G, Durbin R, Genome Project Data Processing S. 2009. The Sequence Alignment/Map format and SAMtools. Bioinformatics 25:2078-9.

41. Li H. 2011. A statistical framework for SNP calling, mutation discovery, association mapping and population genetical parameter estimation from sequencing data. Bioinformatics 27:2987-93. 
42. Nguyen LT, Schmidt HA, von Haeseler A, Minh BQ. 2015. IQ-TREE: a fast and effective stochastic algorithm for estimating maximum-likelihood phylogenies. Mol Biol Evol 32:268-74.

43. Letunic I, Bork P. 2019. Interactive Tree Of Life (iTOL) v4: recent updates and new developments. Nucleic Acids Res 47:W256-W259.

44. Enright AJ, Van Dongen S, Ouzounis CA. 2002. An efficient algorithm for large-scale detection of protein families. Nucleic Acids Res 30:1575-84.

45. Loytynoja A. 2014. Phylogeny-aware alignment with PRANK. Methods Mol Biol 1079:155-70.

46. Alikhan NF, Petty NK, Ben Zakour NL, Beatson SA. 2011. BLAST Ring Image Generator (BRIG): simple prokaryote genome comparisons. BMC Genomics 12:402.

\section{Tables}

Table 1. SNPs resulted unique mutant proteins in BD1 and BD2

\begin{tabular}{|c|c|c|c|c|c|c|c|c|}
\hline SNP & REF & ALT & FrqBD1 & FrqBD2 & p-value & Gene & AA change & Product \\
\hline S1_2609994 & $\mathrm{G}$ & $\mathrm{A}$ & 0 & 105 & $5.61 \mathrm{E}-53$ & nudF_1 & Arg109Cys & ADP-ribose pyrophosphatase \\
\hline S2_266019 & A & G & 0 & 105 & $5.61 \mathrm{E}-53$ & ulaA & Ile354Thr & $\begin{array}{l}\text { Ascorbate-specific permease IIC } \\
\text { component UlaA }\end{array}$ \\
\hline S2_1024884 & G & A & 0 & 105 & $5.61 \mathrm{E}-53$ & putA & Ala600Val & Bifunctional protein PutA \\
\hline S2_989172 & $\mathrm{C}$ & $\mathrm{T}$ & 0 & 105 & $5.61 \mathrm{E}-53$ & yecS & Pro191Ser & YecS \\
\hline S1_798976 & $\mathrm{T}$ & $\mathrm{C}$ & 0 & 105 & $5.61 \mathrm{E}-53$ & suhB & Glu217Gly & Inositol-1-monophosphatase \\
\hline S1_994229 & G & A & 0 & 105 & $5.61 \mathrm{E}-53$ & stcE_2 & Gly201Asp & Metalloprotease StcE precursor \\
\hline S2_921045 & A & $\mathrm{C}$ & 0 & 105 & $5.61 \mathrm{E}-53$ & ctpH_6 & Ile161Ser & $\begin{array}{l}\text { Methyl-accepting chemotaxis } \\
\text { protein CtpH }\end{array}$ \\
\hline S1_1622584 & $\mathrm{G}$ & A & 0 & 105 & $5.61 \mathrm{E}-53$ & cobB & Pro50Leu & $\begin{array}{l}\text { NAD-dependent protein } \\
\text { deacylase }\end{array}$ \\
\hline S2_773493 & $\mathrm{T}$ & A & 0 & 105 & $5.61 \mathrm{E}-53$ & phhA & Gln19Leu & Phenylalanine-4-hydroxylase \\
\hline S1_681574 & G & $\mathrm{T}$ & 0 & 105 & $5.61 \mathrm{E}-53$ & glmM & Arg196Leu & Phosphoglucosamine mutase \\
\hline S2_161094 & $\mathrm{T}$ & G & 0 & 105 & $5.61 \mathrm{E}-53$ & siaT_5 & Ser241Ala & $\begin{array}{l}\text { Sialic acid TRAP transporter } \\
\text { permease protein SiaT }\end{array}$ \\
\hline S1_1452755 & $\mathrm{T}$ & $\mathrm{C}$ & 0 & 105 & $5.61 \mathrm{E}-53$ & cysG_1 & Val38Ala & Siroheme synthase \\
\hline S1_2731709 & G & A & 0 & 105 & $5.61 \mathrm{E}-53$ & $\operatorname{tam} \mathrm{A}$ & Thr266Ile & $\begin{array}{l}\text { Translocation and assembly } \\
\text { module TamA precursor }\end{array}$ \\
\hline S1_545919 & $\mathrm{T}$ & G & 0 & 104 & 4.32E-51 & pctB_1 & Leu249Trp & $\begin{array}{l}\text { Methyl-accepting chemotaxis } \\
\text { protein PctB }\end{array}$ \\
\hline S1_2814292 & $\mathrm{T}$ & $\mathrm{C}$ & 0 & 102 & $4.43 \mathrm{E}-48$ & $\operatorname{argG}$ & Thr283Ala & Argininosuccinate synthase \\
\hline S1_1332186 & $\mathrm{T}$ & G & 0 & 99 & $1.96 \mathrm{E}-44$ & gyrA & Asp660Glu & DNA gyrase subunit A \\
\hline S1_149686 & G & $\mathrm{T}$ & 0 & 99 & $1.96 \mathrm{E}-44$ & murI & Ala137Ser & Glutamate racemase \\
\hline S2_562858 & A & $\mathrm{T}$ & 0 & 99 & $1.96 \mathrm{E}-44$ & $\begin{array}{l}\text { VCA06 } \\
27\end{array}$ & Thr6Ser & rRNA methylase \\
\hline
\end{tabular}




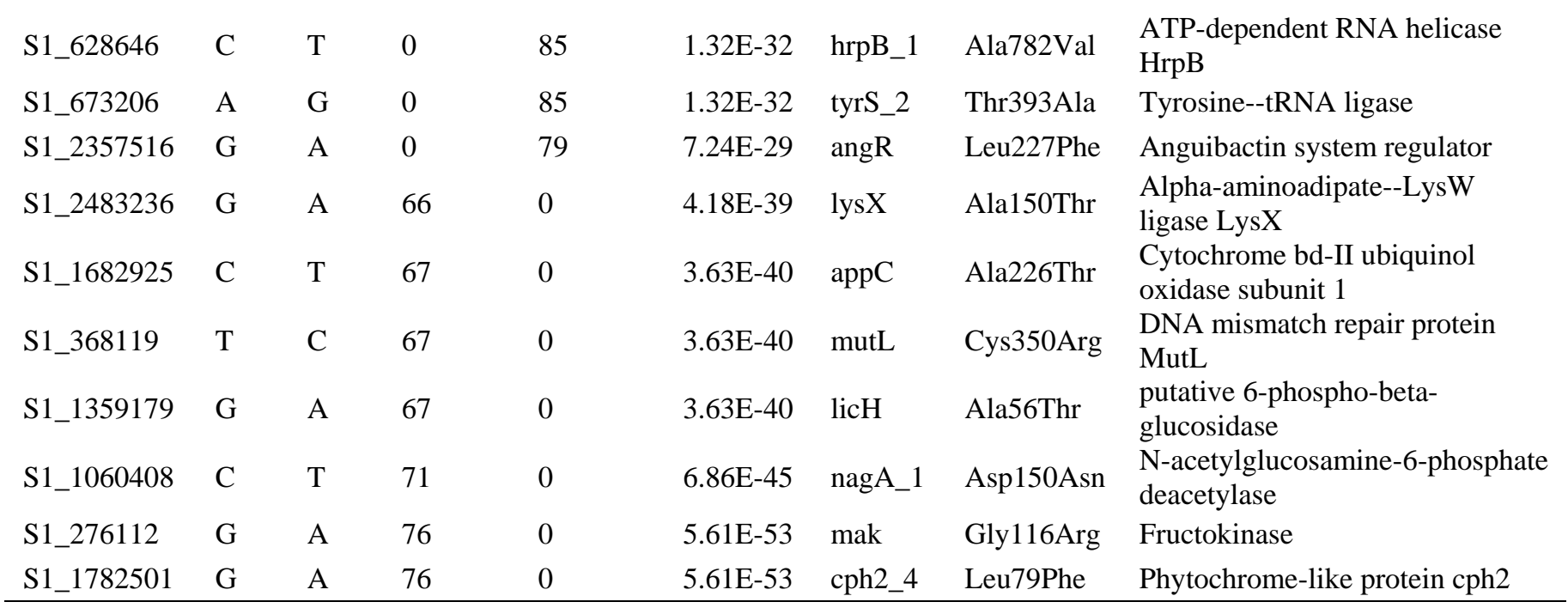

Here, SNP refers the SNPs which had alternative allele uniquely found in more than $80 \%$ of BD1 or BD-2 strains, located within proteins of known functions, and alter amino acid. SNPs were named according its chromosomal position. For example, "S1_2609994" is an SNP/indel site, where "S" stands for site and "2609994" stands for the site's base pair location. Reference allele $=$ REF, alternative allele $=$ ALT, AA change $=$ amino acid change. Freq_BD1 is frequency of alternative allele in BD1 and Freq_BD2 is frequency of alternative allele in BD2. Note that, frequencies of alternative alleles of the SNPs are zero for BD-0. Pvalue is the p-value of Fisher exact test.

\section{Figure Legends}

FIG 1 Phylogenetic analyses of strains showing respective genomic features and year of isolation. (A) Maximum likelihood phylogenetic tree generated from whole genome SNPs and number of isolated $V$. cholerae $\mathrm{O} 1 \mathrm{El}$ Tor strains belonging to lineages BD-0, BD-1, and BD-2 rooted from out-group reference strain Vibrio cholerae N16961. Rings show features of the isolates according to color scheme provided on the left. Tree branches are colored blue, green, and red defining lineages BD-0, BD-1, and BD-2, respectively; (B) Unrooted tree showing independent evolution of BD-1 and BD-2 strains with the number of core genome SNPs of strains in the lineages compared to the N16961 reference strain; and (C) Percentage of isolates per year for the three lineages. Size of the circles indicates percentage of strains belonging to lineages according to the scheme shown.

FIG 2 Box plots of SNPs distribution and indel type in each of three lineage groups. (A) Distribution of 337 synonymous SNP variants. This figure shows that strains of BD-2 lineage accumulated more synonymous SNP variants compared to BD-0 and BD-1 lineages. Notably, synonymous SNP variants do not change the form of protein. (B) Distribution of 613 
582

583

584

585

586

587

588

589

590

591

592

593

594

595

596

597

598

599

600

601

602

603

604

605

606

607

608

609

610

611

nonsynonymous SNP variants. These nonsynonymous SNP variants include 570 missense variants, 38 stop gained variants, 2 splice-region-variants and stop-retained-variants, 2 stop-lost and splice-region-variants, 1 initiator codon variant. (C) Distribution of 348 upstream/downstream SNP variants. (D) Distribution of 238 frameshift indel variants. (E) Distribution of 107 upstream/downstream indel variants. (F) Distribution of 68 indel variants, including 13 conservative-inframe-insertions, 14 disruptive-inframe-insertions, 11 frameshiftvariant and stop-gained, 10 disruptive-inframe-deletions, 10 conservative-inframe-deletions, 1 stop-gained and disruptive-inframe-deletions, 2 feature-elongations, 1 frameshift-variant and stop-lost and splice-region-variant, 1 stop-gained and disruptive-inframe-insertion, 2 frameshiftvariant and splice-region-variant, 2 frameshift-variant and start-lost, 1 stop-gained and conservative-inframe-insertion.

FIG 3 SNP analysis of genetic diversity. (A) Phylogenetic tree map of the strains and heat map for genotypes of 140 SNPs significantly associated with different lineages. Colors used delineate four different nucleotides where white represents the missing genotype. Heatmap shows clear differences in the lineages. (B) Number of core genome SNPs referencing the year of isolation. The figure shows steady accumulation of SNPs of different lineage strains over time. (C) Regression analysis of root-to-tip distance for strains of the lineages. This figure shows diversity of strains of different lineages. (D) Miami plot of alternative allele frequencies of SNPs for the dominant lineages BD-1 and BD-2. This figure shows the clear difference in SNP accumulation by the two dominant lineages BD-1 and BD-2.

FIG 4 Pangenome analysis showing differences in abundance of gene clusters among the lineages. (A) Relative gene abundance of lineages identified by Roary. Features of the sequences are shown with bars and details for features listed in Table S1. (B) BLAST coverage of SXT regions of BD-1 isolates compared with ICE-GEN. Rings represent sequentially outwards following Table S1. Outermost ring shows the different genes of ICE-GEN. (C) BLAST coverage of SXT regions of BD-2 isolates compared with ICE-TET. The rings represent strains of BD-2 sequentially outwards following Table S1. The outermost ring shows different genes of ICE-TET.

FIG 5 Schematic diagram of VSP-II. Schematic alignment view of VSP-II regions for the isolates. Direction of gene transcription is indicated by arrows and gene shadows represent 
612 functional annotation. Six types were identified with all BD-0 strains wild-type VSP-II. Two

613 major types, var-2 and var-3, observed for most BD-2 strains and one major type var-4 for most

614 BD-1 strains.

\section{Supplementary Information}

616 FIG S1 Bayesian phylogenetic analysis of $\boldsymbol{V}$. cholerae 01 . Node ages obtained from BEAST

617 analysis. Tree visualized using FigTree v1.4.4. Colors of clades reference the lineage.

FIG S2 Manhattan plots of $p$-values for association studies of SNPs and BD-1 and BD-2

lineages. Blue represent suggested significant and red indicates high significance. Association analysis reveals 140 SNP difference between BD-1 and BD-2 lineages.

FIG S3 Study flow chart. Data curation and analyses steps are given in the flow chart.

Table S1. Genetic characteristics of strains included in the study. Lineage refers to ctxB allele, and PLE are tabulated.

Table S2. Number of strains belonging to the different lineages. Here, N_BD-0 = number of strains belonging to BD-0; N_BD-1 = number of strains belonging to BD-1; and N_BD2 = number of strains belonging to BD-2.

Table S3. Fisher exact test identifying significantly associated 140 SNPs and 31 indels of the

by Fisher exact test. SNPs and indels named according to chromosomal position. For example, value is Fisher exact test value. Variant type indicates SNP and indel type. 
636 Gene cluster refers to group of genes clustered based on existence in the strains of different

637 lineages. Code for number of genes in lineage BD-0, BD-1, and BD-2 is N_BD-0, N_BD-1, and

638 N_BD-2, respectively.

639 Table S5. Common and unique genes of the different lineages. (A) Genes detected in more

640 than $95 \%$ of BD-0, BD-1, and BD-2 strains. (B) List of unique genes in BD-2. (C) List of unique

641 genes in BD-1.

642 Table S6. PanGWAS identified lineage associated genes. (A) List of genes associated with

643 BD-0 and BD-1. (B) List of genes associated with BD-0 and BD-2. (C) List of genes associated

644 with BD-1 and BD-2.

645 Table S7. Number of strains with phage inducible chromosomal island like elements (PLE).

646 Absence of PLE $=$ PLE(-), and PLE1 and PLE2 are two different types of PLE. 
bioRxiv preprint doi: https://doi.org/10.1101/2021.12.16.473094; this version posted December 17, 2021. The copyright holder for this preprint (which was not certified by peer review) is the author/funder, who has granted bioRxiv a license to display the preprint in perpetuity. It is made available under aCC-BY-ND 4.0 International license.

(A)

1. Year of Isolation

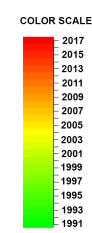

2. VSP-II Type

$\square$ Wild type (N16961)

var. 1 ( $\triangle V V_{-}$4955-VC_498)

var. 2 ( $\left.\Delta V C \_495-V C \_500\right)$

$\square$ var. 3 ( $\Delta V C_{-}$491-VC_501)

- var. 4 ( $\Delta V V_{-}$4955-VC_512)

var. 5 ( $\left.\Delta V C_{-} 490-V C \_516\right)$

3. VPII TYPE

Wild Type (N16961)

variant (VC_819- $\Delta$ VC_821)

4. ctxB genotype

$\square \operatorname{ctxB1}$

$\operatorname{ctxB3}$

$\mathrm{ct} \times \mathrm{B} 7$

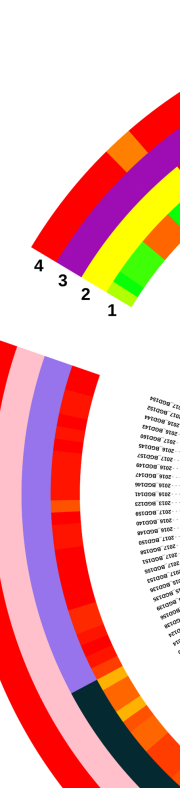

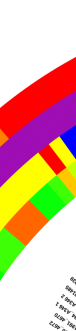
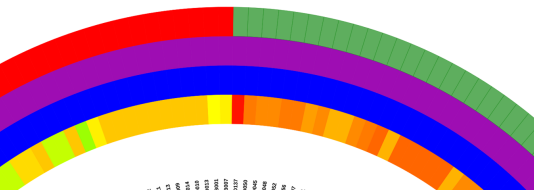

$+n^{2}$

11)

Wn In

है

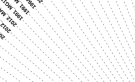

\begin{tabular}{r}
\hline$\times$ \\
+ \\
\end{tabular}
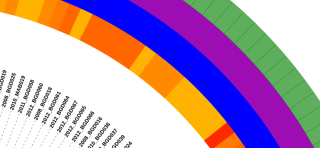

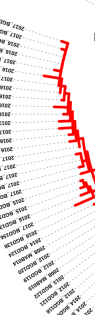
${ }^{216961}$ BD
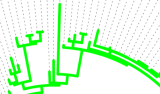
4 3
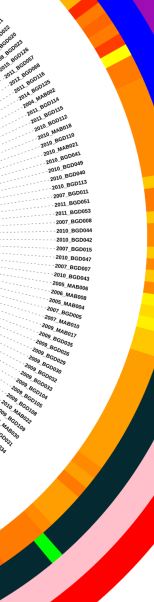

(B)

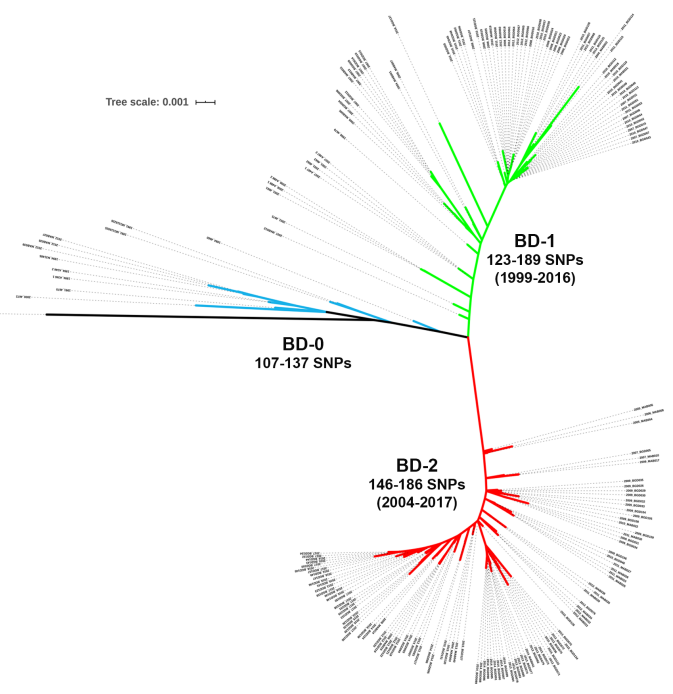

(C)

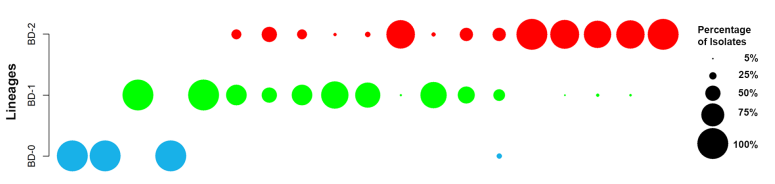

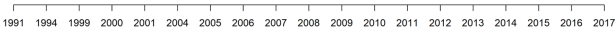
Year of Isolation 
(A)

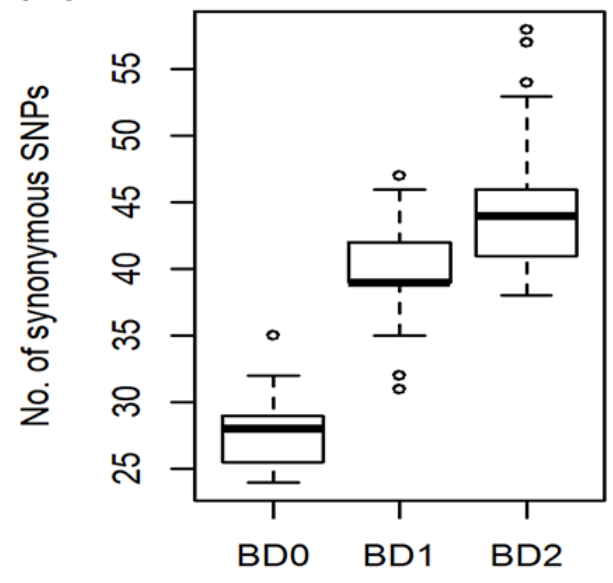

Lineage

(D)

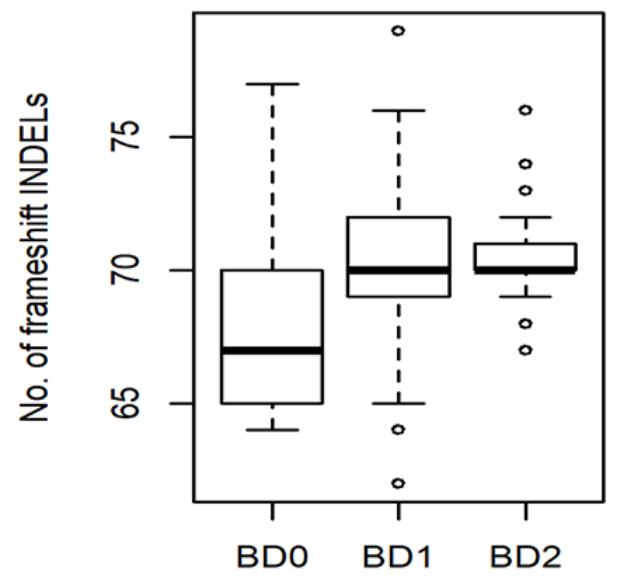

Lineage
(B)

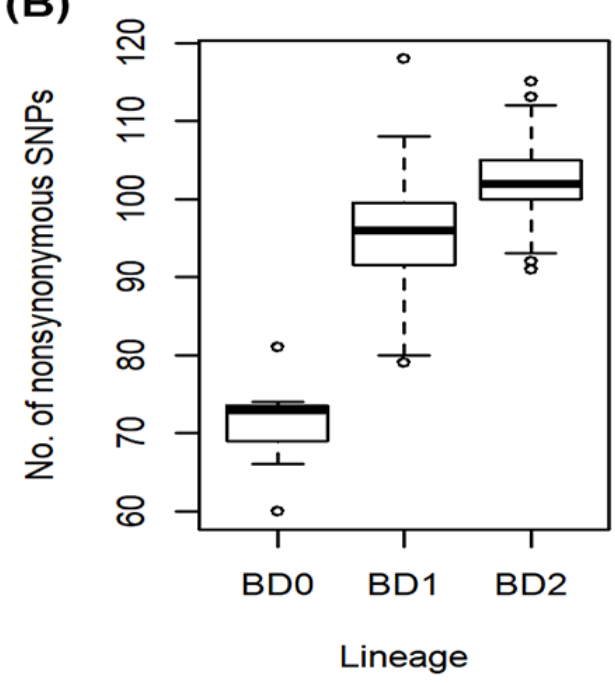

(E)

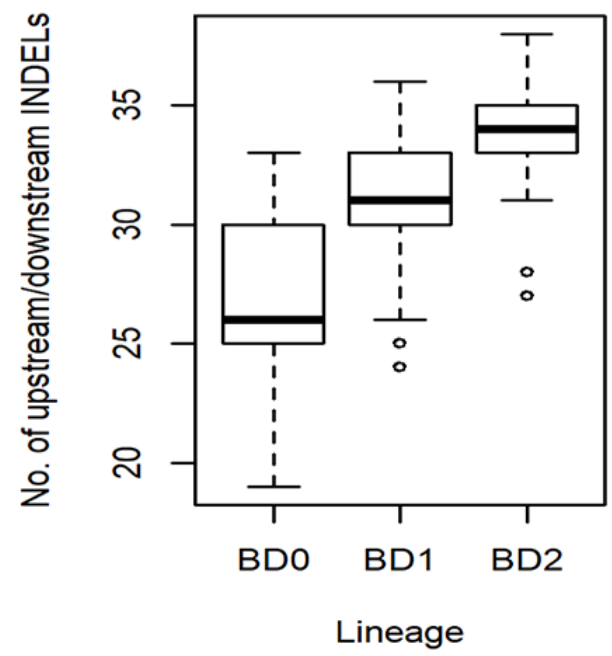

(C)

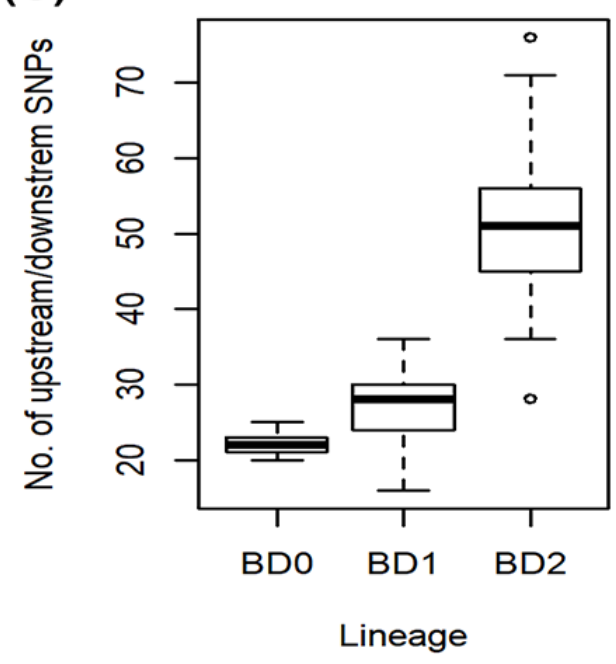

(F)

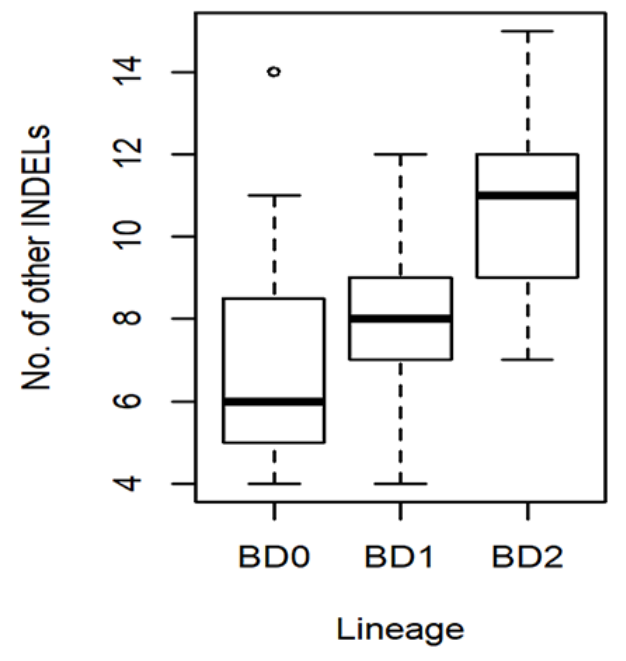


(A)

bioRxiv preprint doi: https:/doi.org/10.1101/2021.12 16.473094 * this version posted December 17, 2021. The copyright holder for this preprint

(which was not certified by peer review) is the author/funder, who has granted diviv a license to display the preprint in perpetuity. It is made

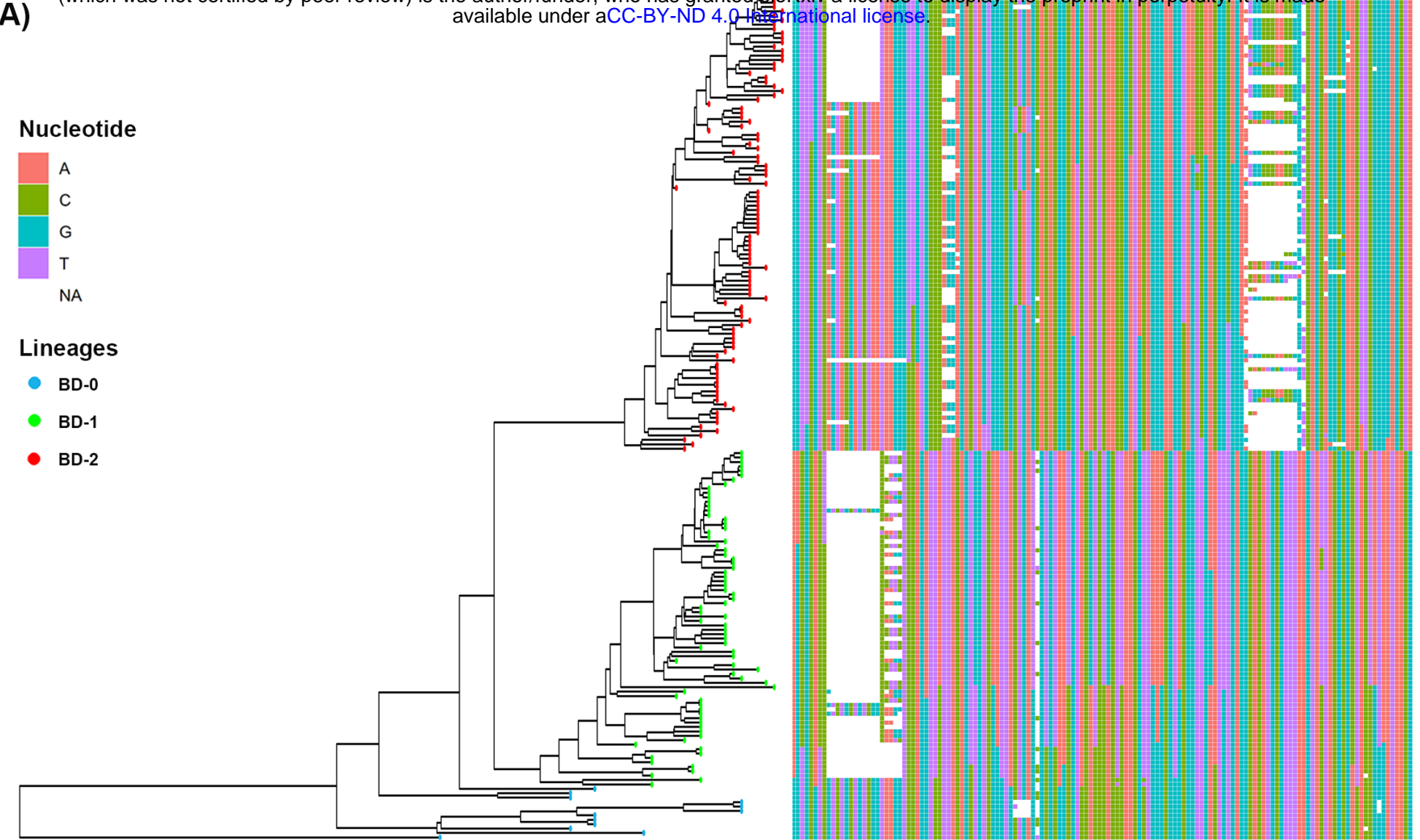

140 SNPs significantly associated with lineages

(B)

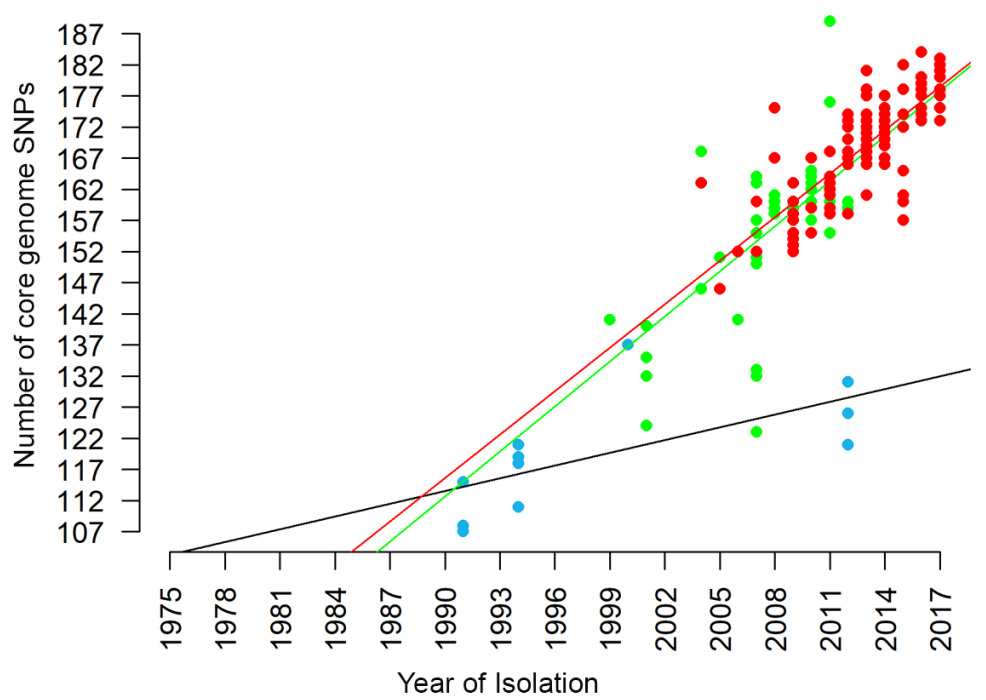

(C)

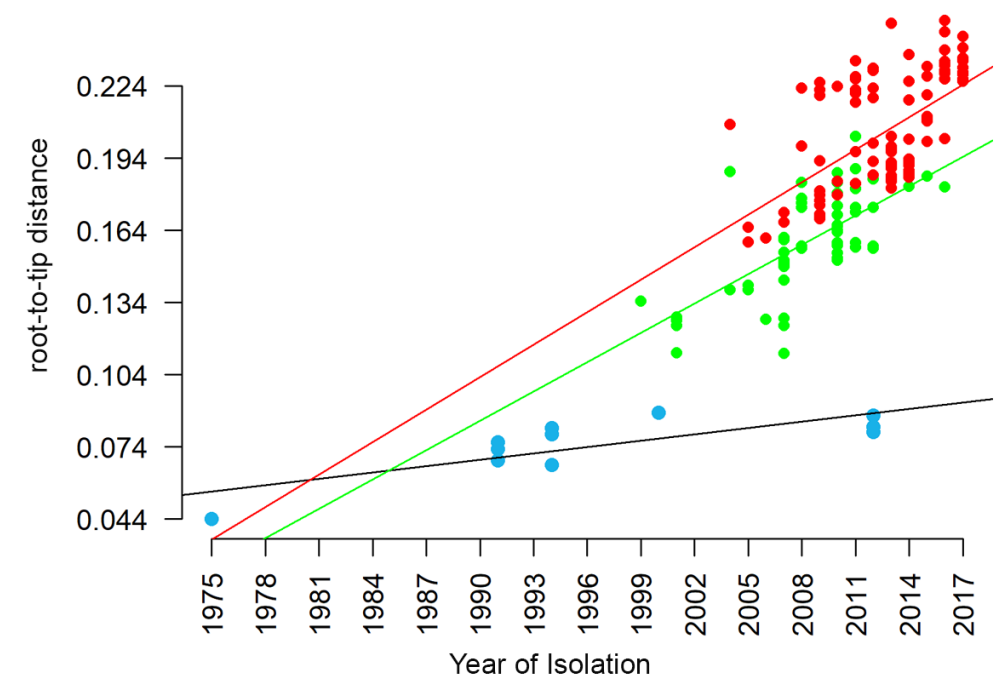

(D)

- SNP variant with frquency $>0.5$
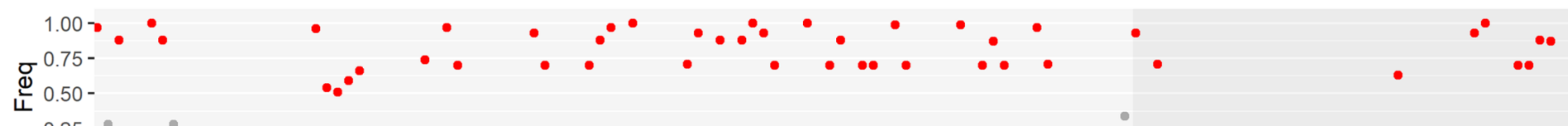

BD-1

$0.25-$

Chromosome-2

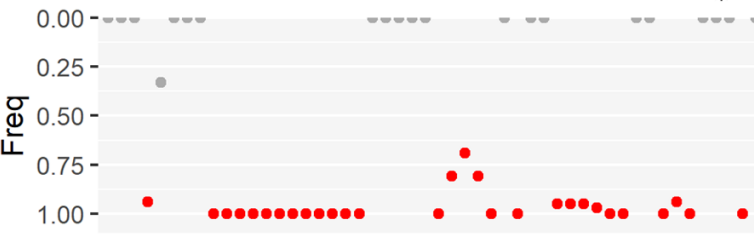


bioRxiv preprint doi: https://doi.org/10.1101/2021.12.16.473094; this version posted December 17, 2021. The copyright holder for this preprint (which was not certified by peer review) is the author/funder, who has granted bioRxiv a license to display the preprint in perpetuity. It is made available under aCC-BY-ND 4.0 International license.

(A)
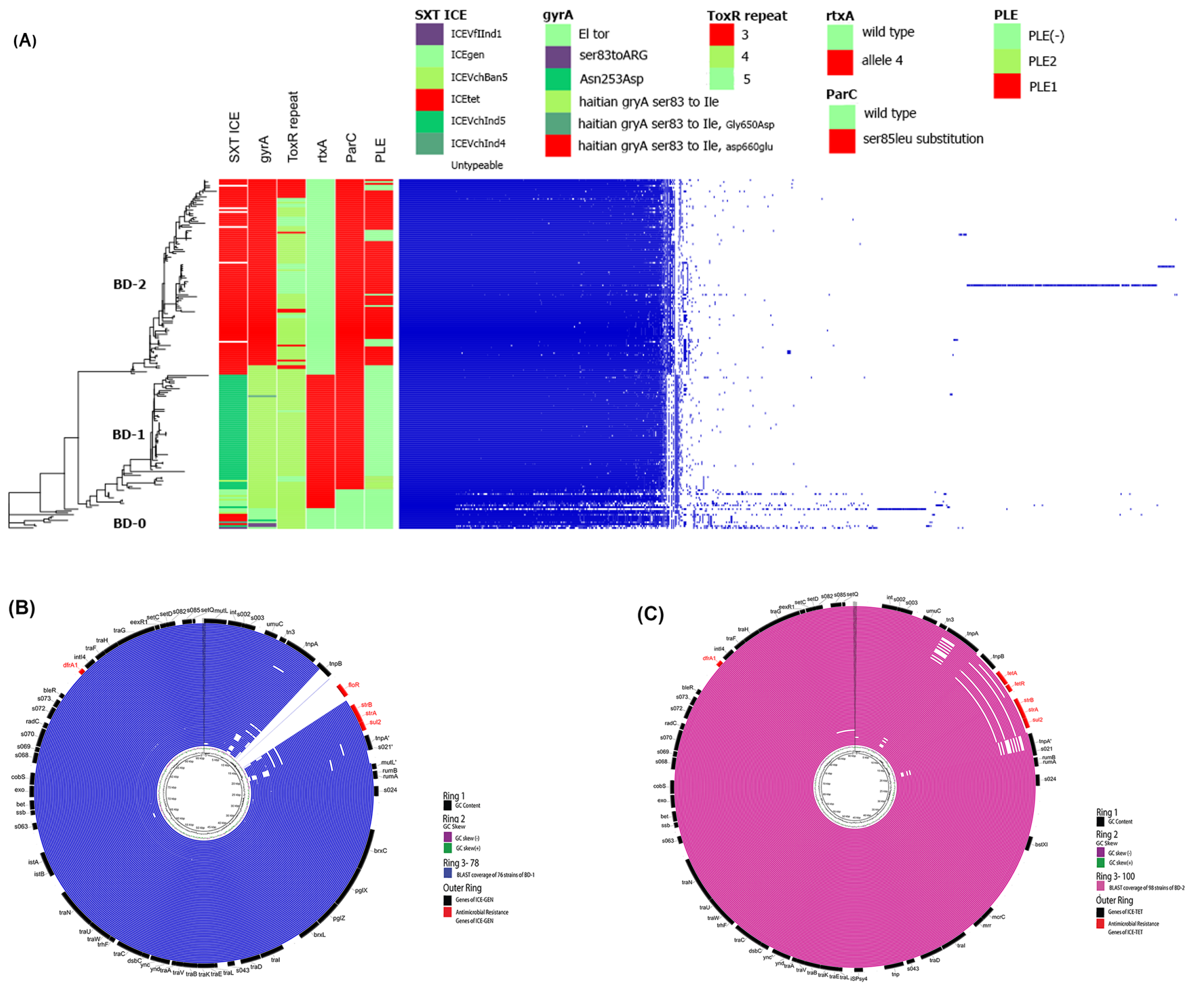
bioRxiv preprint doi: https://doi.org/10.1101/2021.1216.473094: this version posted December 17, 2021. The copyright holder for this preprint (which was not certified by peer review) is the author/funder, who has granted bioRxiv a license to display the preprint in perpetuity. It is made available under aCC-BY-ND 4.0 International license.

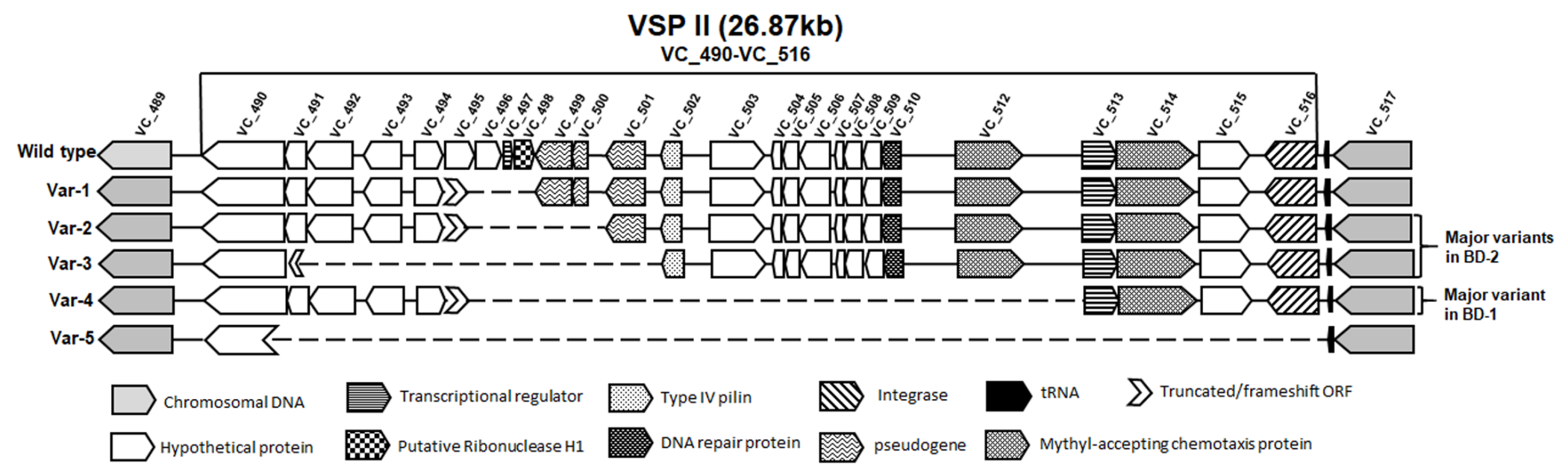

